\title{
Process of neovascularisation compared with pain intensity in tendinopathy of the long head of the biceps brachii tendon associated with concomitant shoulder disorders, after arthroscopic treatment. Microscopic evaluation supported by immunohistochemical markers of the endothelial cells
}

\author{
J. Zabrzyński ${ }^{1}$, Ł. Paczesny², Ł. Łapaj ${ }^{3}$, D. Grzanka ${ }^{4}{ }^{5}$, J. Szukalski ${ }^{6}$ \\ ${ }^{1}$ Department of Orthopaedic Surgery, Multidisciplinary Hospital, Inowroclaw, Poland \\ ${ }^{2}$ Department of Orthopaedic Surgery, Orvit Clinic, Torun, Poland \\ ${ }^{3}$ Department of General Orthopaedics, Musculoskeletal Oncology and Trauma Surgery, \\ Poznan University of Medical Sciences, Poznan, Poland \\ ${ }^{4}$ Department of Clinical Pathomorphology, University Hospital No. 1, Bydgoszcz, Poland \\ ${ }^{5}$ Department of Dermatology, Sexually Transmitted Infections and Immunodermatology, \\ Nicolaus Copernicus University, Bydgoszcz, Poland \\ ${ }^{6}$ Division of Pathology, Multidisciplinary Hospital, Inowroclaw, Poland
}

[Received: 6 June 2017; Accepted: 4 September 2017]

Background: Tendinopathy of the long head of the biceps brachii tendon (LHBT) is one of the most common, painful conditions of the anterior part of the shoulder and often coexists with rotator cuff tears. Multifactorial aetiopathology of tendinopathy is poorly understood; however, several studies indicated that it is seen predominantly in areas with decreased vascularity of the tissue; the pathology is also characterised by expansive and abundant neovascular in-growth. The aim of the study was to investigate the relationship between the neovascularisation of proximal part of the LHBT and pain along the bicipital groove.

Materials and methods: Tissue material was obtained from 28 patients who underwent a shoulder arthroscopy and experienced pain along the bicipital groove measured using Visual-Analog Scale (VAS) score. CD31 and CD34 molecules were visualised by immunohistochemical method to assess biceps tendon neovascularisation and quantify it based on a Bonar scoring system.

Results: Although all patients reported pain prior to arthroscopy (mean VAS score was 7.5), microscopic examination did not reveal neovascularisation in all cases. Immunohistochemical staining for CD31 and CD34 allowed for very precise visualisation and quantification of neovascularisation; however there was also no correlation between vessels in-growth scores and pain

Conclusions: The obtained data suggest that neovascularisation process in tendinopathy is not directly related to pain; however, further studies are needed to explain its significance in the LHBT tendinopathy. (Folia Morphol 2018; 77, 2: 378-385)

Key words: neovascularisation, biceps tendinopathy, tendinopathy, CD31, CD34, immunohistochemical

Address for correspondence: J. Zabrzyński, MD, Multidisciplinary Hospital, Department of Orthopaedic Surgery, ul. Poznańska 97, 88-100 Inowrocław, Poland, tel/fax: +48 5235454 78, e-mail: zabrzynski@gmail.com 


\section{INTRODUCTION}

Tendinopathy of the long head of the biceps brachii tendon (LHBT) is one of the most common causes of chronic anterior shoulder pain, typically localised within the bicipital groove on the proximal part of humerus, $3-6 \mathrm{~cm}$ below the anterior margin of the acromion [17, 18, 38]. Up to $90 \%$ of cases of the LHBT tendinopathies are accompanied by additional shoulder disorders, such as massive rotator cuff tears (RCTs); in numerous cases treatment of this condition is difficult $[3,12]$.

The aetiopathology of the LHBT tendinopathy is complex and not fully understood. One of most widely accepted theories of its development focuses on the vascular origin of the process. This theory is based on the assumption that a gradual degradation of tendons occurs predominantly in hypovascular or avascular regions, which are prone to subsequent hypoxia. This is supported by data from the literature, which indicates that certain tendons tend to fail in hypovascular or critical zones e.g. Achilles tendon, rotator cuff tendons, tibialis posterior tendon [13, 31, 33]; similarly, in the LHBT a hypovascular area was demonstrated about $1-3 \mathrm{~cm}$ from its origin [12].

The degradation of tendinous tissue leads to significant changes in the histology of tendon. Microscopic findings include alterations in fibre alignment, cellular structure and collagen stain ability, as well as increased vascularity. Several facts suggest that the last aforementioned conditions could play an important role in mediation of pain related to the LHBT tendinopathy, where the vascular network tends to become substantially denser [8, 21, 34]. Newly formed vessels are followed by nerves, which usually arrive from the supraglenoid tubercle; their endings may contain increased levels of calcitonin gene-related peptide (CGRP) and substance $P$ which are likely responsible for pain sensation [5].

Surprisingly, despite recent advances in imaging of the LHBT tendinopathy and the development of therapeutic strategies, relatively few studies have tried to quantify histological changes related to this condition. In most studies the evaluation has been based on semiquantitative Movin and Bonar scores, which require special staining protocols (GAG) and focus on several microscopic features of cells and extracellular matrix. Interestingly, despite the fact that neovascularisation seems to play an important role in the development of pain related to LHBT tendinopathy, few studies have investigated the extent of this phenomenon or have tried to explain how it is related to clinical symptoms.

In recent years multiple publications have documented that two markers, CD31 and CD34, can be successfully used to visualise presence of newly formed vessels, especially in various types of tumours. CD31 is a $130 \mathrm{kDa}$ trans-membrane glycoprotein present on the surfaces of: platelets, monocytes, macrophages, neutrophils, is a constituent of the endothelial intracellular junction and plays a role in adhesion of endothelial cells (EC) and in the angiogenic process [29]. CD31 is expressed in $90 \%$ of EC tumours - it is generally regarded as the best single marker of EC differentiation. CD34 is a 110 kDa transmembrane glycoprotein present on leukemic cells, EC, and stem cells; it plays a role in angiogenesis and is considered a marker of vascular tumours [29].

Several authors suggested that these markers could aid in the diagnosis of non-vascular tumours and pathological neovascularisation [11, 27-29, 35]. That is why we conducted a study which could demonstrate (1) whether it is possible to clearly visualise neovascularisation of the LHBT using CD31 and CD34 markers, (2) if there is a relationship between the extent of LHBT neovascularisation and patient reported clinical symptoms.

\section{MATERIALS AND METHODS}

This study included 28 consecutive patients who underwent shoulder arthroscopy and were diagnosed, among other pathologies, with the LHBT tendinopathy. The study was approved by local bioethics committee (approval no. KB 598/2016). All patients were volunteers and gave informed consent. We included patients who were diagnosed based on clinical examination (unilateral pain during palpation of the anterior part of the humerus, in the area of the bicipital groove; the minimal duration of symptoms was 3 months), sonographic studies or non-contrast magnetic resonance scans. In most cases the LHBT tendinopathy was associated with lesions of the rotator cuff (19 patients); however, we did not include patients with rheumatic diseases, previous surgical treatment or corticosteroid injections in the past 12 months. The mean age of the subjects was 52 years (28-74 years; standard deviation [SD] 10.5); the cohort included 13 males and 15 females. All patients underwent physical examination prior to surgery: anterior shoulder pain was evaluated using a VisualAnalog Scale (VAS) with a scale from 0 (no pain) to 10 , 
(most severe pain). Additionally, we performed clinical test - tenderness over the bicipital groove.

Since obtaining samples of healthy LHBT tendons is problematic, as a negative control group we included 4 patients who underwent anterior cruciate ligament $(A C L)$ reconstruction using hamstring tendons (semitendinosus, semimembranosus and biceps femoris muscles) [36]. From these patients we obtained fragments of the semitendinosus tendons which were discarded during preparation of the ACL graft. In order to confirm proper staining results of immunohistochemical studies, we also included positive controls - slides obtained from paraffin blocks which contained samples of 2 vascular tumours. These samples were confirmed to contain CD31/CD34 immunopositive cells using a different batch of antibodies than used in this study.

Long head of the biceps brachii tendon samples were obtained during shoulder arthroscopies which was performed in the beach chair position in general anaesthesia. During the procedure, the LHBT was inspected for pathology and subsequently subjected to tenodesis or tenotomy. Next, the intra-articular part of the tendon was removed using arthroscopic scissors; the resection started about $1 \mathrm{~cm}$ from the origin at the supraglenoid tubercle and ended in the bicipital groove area. All obtained tissue fragments were immediately fixed in $10 \%$ buffered formalin; the remaining proximal part of the tendon was then vaporised.

All tissue samples were dehydrated in increasing concentrations of ethanol, cleared in xylene, embedded in paraffin and cut into $4 \mu \mathrm{m}$ thick serial, longitudinal sections which were mounted on glass slides. One sample from each patient was stained using haematoxylin and eosin (H\&E) procedure for routine histopathological examination, using optical microscopy. Subsequently, sections were stained using antibodies for CD31 (Roche, 760-4378, Sierra College Boulevard Rocklin, California, USA), and CD34 (Roche, 790-2927, Hague Road, Indianapolis, USA). Briefly, sections were deparaffinised and rehydrated; endogenous peroxidase was blocked with 3\% hydrogen peroxide. Next, samples were stained using a fully automated Ventana BenchMark GX (Rotkreuz, Switzerland) according to manufacturer's instructions; incubation with primary antibodies took $40 \mathrm{~min}$.

The assessment of neovascularisation was based on the criteria from the Bonar scale $[20,22,36]$. The scores $0,1,2,3$ correspond to the following stages of neovascularisation seen in 10 high-power fields (HPFs): 0 - modest and average occurrence of the blood vessels, 1 - incidental cluster of capillaries less than 1 per 10 HPFs, 2 - indicates 1-2 clusters per 10 HPFs, 3 - indicates more than 2 clusters per 10 high-power fields. In each patient we analysed ten high-magnification fields which demonstrated presence of the densest capillary network.

\section{Statistical analysis}

Data were statistically analysed using Statistica 12 software (Statsoft, Krakow, Poland). Normality of data distribution was evaluated using Saphiro-Wilk test; correlation analysis was performed using Spearman's test.

\section{RESULTS}

\section{Patient characteristics}

All patients included in this study reported anterior shoulder pain, which was moderate to severe (range 5-9 points), with a mean VAS score of 7.5 (SD 0.92). Three patients reported presence of anterior shoulder pain at night. A preoperative physical examination showed that palpation in the bicipital groove was painful in 26 patients (93\%, 14 women and 12 men). The bicipital groove tenderness test was negative in 2 patients - one of them had a totally ruptured LHBT, in the other shoulder the tendon was not ruptured and the patient had moderate overall shoulder pain with VAS score of 5 . During shoulder arthroscopy the LHBT tendinopathy was confirmed in all cases, mostly accompanied by other lesions: RCTs were seen in 19 patients, subacromial impingement in 16 patients, labral defects in 2 patients. In 6 cases the LHBT lesion was treated with tenotomy, in 21 cases with tenodesis. There was one complete LHBT rupture which required the intra-articular stub resection.

Macroscopic assessment of the removed LHBT portions revealed several pathological features, among all of them: tears, widening and flattening of the resected tendon part.

\section{Microscopic evaluation of tendon samples}

Microscopic evaluation of H\&E stained slides showed advanced degeneration among all of the specimens; neovascularisation was observed in 22 (78\%) patients (Fig. 1B, C). We graded the neovascularisation process, present in 22 specimens, according to the criteria adopted from the Bonar scale. Grade 0 was present in 6 samples. Grades 1 and 2 were 


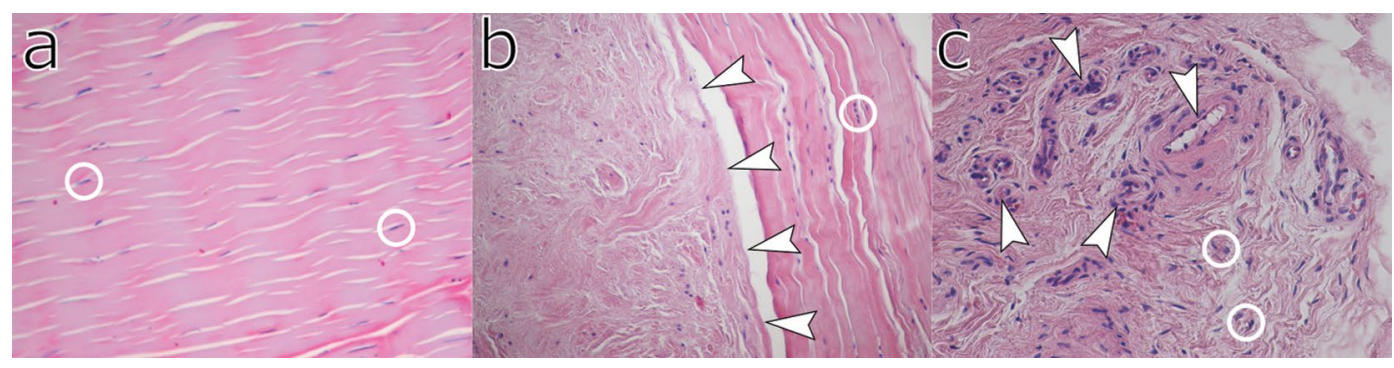

Figure 1. Structure of normal and pathological tendon samples; haematoxylin and eosin staining. A. Normal tendon with wavy configuration of fibres and spindle-shaped nuclei (circles); magnification 100x; B. Long head of the biceps brachii tendon (LHBT) tendinopathy — disorganised fibrous structure visible in damaged areas (arrows), round nuclei (circles) typically seen in pathological tenocytes; magnification 100×; C. Neovascularisation in LHBT tendinopathy: a network of randomly scattered capillaries (arrows) is present among disorganised extracellular matrix; rounded nuclei of tenocytes are visible (circles). Solitary capillary vessels are forming organised groups of new vessels in the tendon tissue, called clusters; magnification $200 \times$.

Table 1. Distribution of pathological alterations among 28 patients

\begin{tabular}{|c|c|c|c|c|c|c|c|c|c|c|c|c|c|c|c|c|c|c|c|c|c|c|c|c|c|c|}
\hline Patient number & 1 & 2 & 3 & 4 & 5 & 6 & 7 & 8 & 9 & 10 & 111 & 12 & 131 & 141 & 1516 & 1617 & 718 & 19 & 20 & 212 & 222 & 232 & 242 & $25 \quad 26$ & 627 & 728 \\
\hline $\begin{array}{l}\text { Visual-Analog } \\
\text { Scale (VAS) score }\end{array}$ & 8 & 7 & 8 & 5 & 6 & 7 & 6 & 7 & 8 & 7 & 7 & 8 & 8 & 8 & 8 & 7 & 8 & 7 & 7 & 8 & 7 & 8 & 9 & 9 & 7 & 9 \\
\hline $\begin{array}{l}\text { Neovascularisation } \\
\text { level according to } \\
\text { Bonar score }\end{array}$ & 2 & 2 & 3 & 0 & 1 & 1 & 0 & 0 & 1 & 3 & 2 & 3 & 2 & 3 & 3 & 0 & 2 & 1 & 3 & 2 & 2 & 0 & 1 & 2 & 3 & 0 \\
\hline
\end{tabular}

identified in 5 and 8 samples, respectively. The highest grade - 3, was present in 9 samples and it was the most numerous group (Table 1, Fig. 2). In cases where neovascularisation was present, vessels were uniformly scattered in the tendon tissue, except for 2 cases, where we noted formation of angio-fibrous granulation with massive formation of new vessels (Fig. 3).

Immunohistochemical staining of vessels from LHBT samples using CD31 and CD34 antibodies demonstrated a nearly identical profile of both antigens in all specimens (Fig. 4C, D). Both markers provided excellent visualisation of capillaries' walls in tendon tissue as well as positive controls, allowing a precise quantification of neovascularisation process; negative controls did not exhibit any unspecific reactions or artifacts and contained very few capillaries (Fig. 4A, B). There were no differences between scores obtained after neovascularisation assessment in slides stained using CD31 and CD34 antibodies.

\section{Statistical analysis}

Statistical analysis demonstrated that there was no correlation between VAS scores and the extent of neovascularisation quantified in this study (Spearman's coefficient $=0.233$; significance level, $p=0.2323$ ).
Six patients, with the absence of neovascularisation in samples, had anterior shoulder pain (VAS score 5-9). Additionally, in 5 of them the bicipital groove tenderness test was positive (Table 1). All patients with neovascularisation process of the LHBT demonstrated anterior shoulder pain (VAS score 6-9), interestingly one of these patients had a complete rupture of the tendon and did not demonstrate tenderness over the bicipital groove. Despite that fact, permanent painful disorders were present in the anterior area of the shoulder (VAS score 8).

\section{DISCUSSION}

In healthy tendons vascularisation is modest with a small number of capillaries located between bundles. Vessels of the tendon arise mainly from the musculotendinous junction, osteotendinous junction and connective tissue sheath [13]. Vessels are arranged mainly longitudinally to the long axis of the tendon. Tendons are metabolically active tissues and demand adequate blood distribution [1]. Tendon's nutrition depends on synovial fluid delivery and blood supply from local vessels. Despite this, tendons are characterised by a low vascular perfusion.

The process of neovascularisation occurs in pathological condition in tendons such as osteoarthritis, 


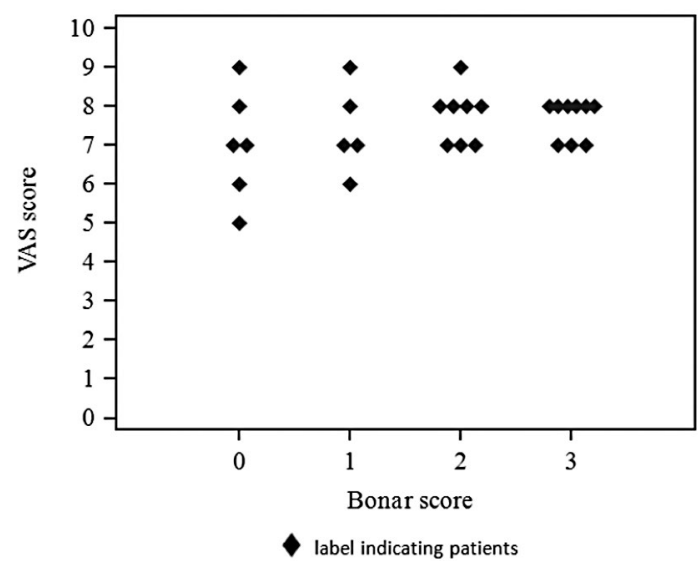

Figure 2. Comparison of neovascularisation process and pain intensity. Scatter plot showing relationship between neovascularisation and painful disorders according to Visual-Analog Scale (VAS) score among patients.

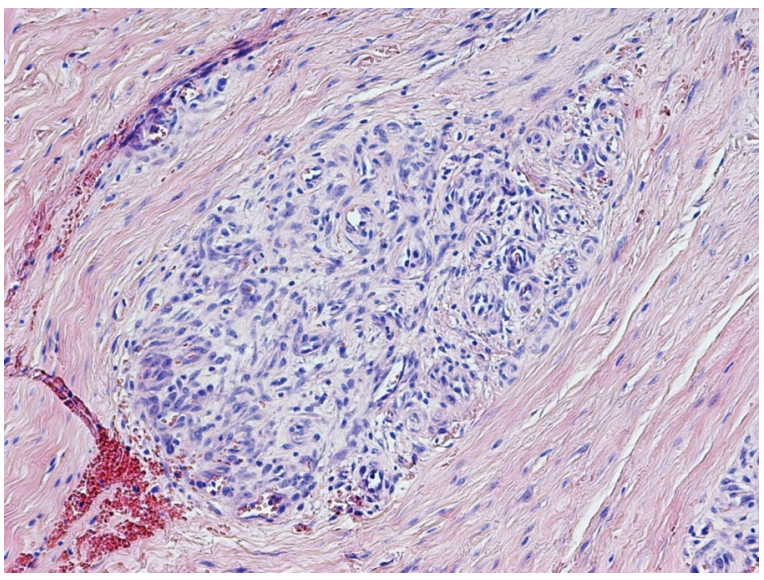

Figure 3. Extraordinary, abundant formation of new vessels in the long head of the biceps brachii tendon. Staining haematoxylin and eosin; magnification $100 \times$.

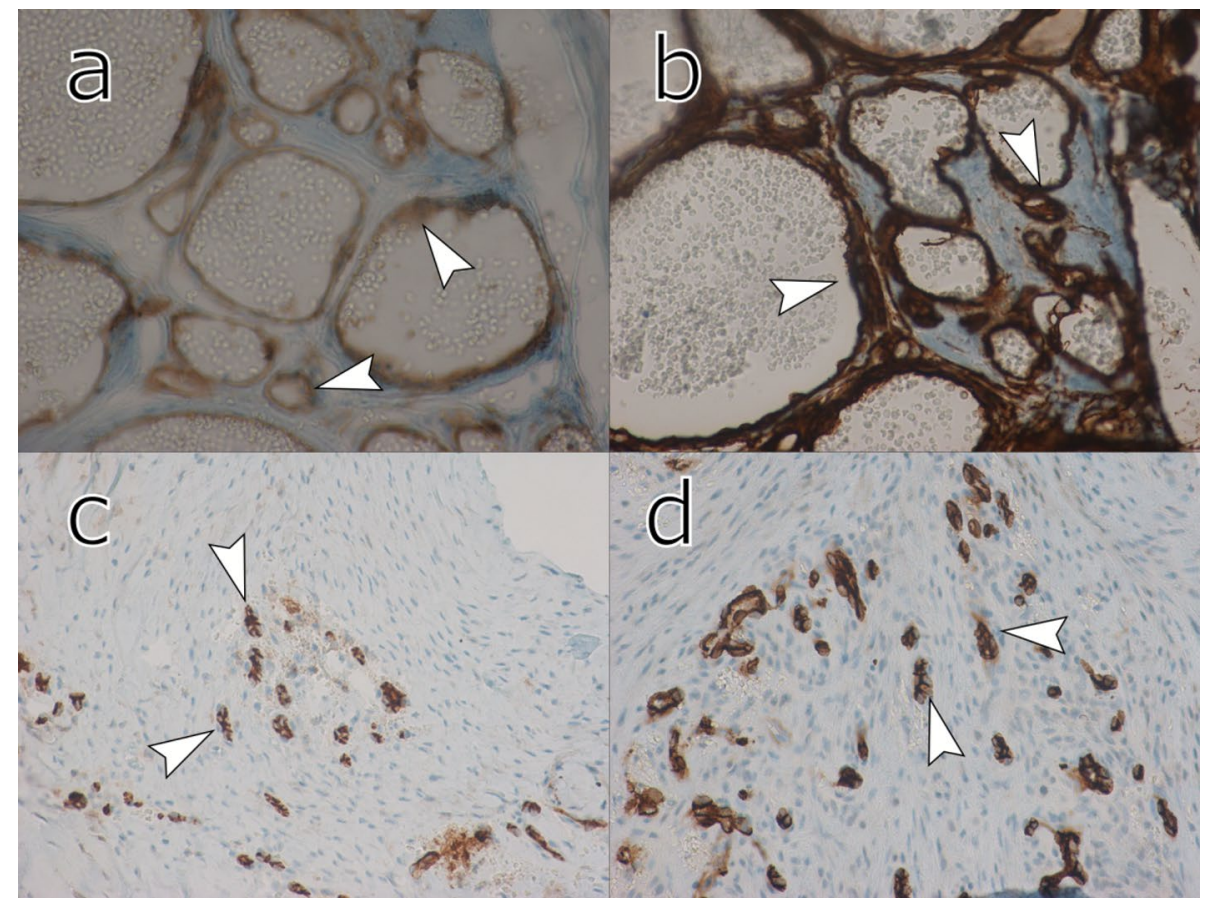

Figure 4. Immunohistochemical examination of neovascularization. A. CD31 — positive control, Hemangioma capillare simplex — arrows indicate positive reaction which corresponds to vascular walls; magnification $200 \times$; B. CD34 — positive control, Hemangioma capillare simplex - arrows indicate positive reaction corresponding to vascular wall; magnification 200 $;$; C. CD31 staining — long head of the biceps brachii tendon (LHBT) tendinopathy; arrows indicate expression of newly formed capillaries arranged in a random pattern; magnification $200 \times$; D. CD34 staining - LHBT tendinopathy, arrows indicate expression pattern of randomly scattered capillaries in disorganised extracellular matrix; magnification $200 \times$.

retinopathy, inflammation, tumours. Presence of neovascularisation is well documented in advanced tendinopathies and can be demonstrated using Doppler ultrasound examination $[9,15,19,20,26,30]$. Similarly, in our study the presence of neovascularisa- tion of the LHBT was confirmed in $78 \%$ of specimens. In recent studies, microdialysis techniques were used to obtain the extra-cellular fluid from tendons and examine presence of mediators associated with neovascularisation [1]. High levels of lactate concentration 
were found in tendons of patients suffering from tendinopathy, which indicates insufficient vascular supply and is associated with anaerobic metabolism [4]. Hypoxia leads to increased synthesis of vascular endothelial growth factor (VEGF), which is an important angiogenesis regulator and has an influence on tendon's proteases activity [1]. This is accompanied by increased synthesis of matrix metalloproteases, which degrade tendons structure to facilitate expansion of new vessels. VEGF level is also elevated during the regeneration of tendon tissue, after the inflammatory phase, during formation and remodelling phases [24]. Interestingly, recent studies showed that other forms of mechanical stimuli such as massage, can also initiate structural changes in tendons and muscles. Using a rat model, Andrzejewski et al. [6, 7] demonstrated that short-term massage can up regulate VEGF in skeletal muscles, while long-term massage can induce structural changes in the structure of collagen fibres. Other animal studies of tendons subjected to acute injuries, demonstrated presence of neovascularisation in such cases, which suggests that it is associated with healing response $[23,32]$. Since it is difficult to obtain such specimens from patients with fresh tendon injuries, most publications focus on chronic injuries $[9,15,26,39]$. These reports indicated that despite formation of new vessels, there is no healing response. Instead, most authors reported accumulation of new vessels accompanied by a network of altered tenocytes unable to maintain the organised structure of the extracellular matrix [40].

Isolated pathology of the LHBT is relatively rare and seen predominantly in young throwing sports athletes. In most cases it affects non-athletes and coexists with overuse trauma and other shoulder pathologies, such as subacromial impingement, RCTs, superior labral tear from anterior to posterior [16, $37,38,40]$. Consequently it was suggested that anterior shoulder pain may not result strictly from LHBT pathology but is rather a manifestation of complex shoulder lesions [36]. Still, the role of the LHBT in anterior shoulder pain is undeniable, since arthroscopic tenotomy and tenodesis procedures usually result in pain relief $[17,36]$.

This is consistent with observations from our study, where vessels formation was not directly associated with painful condition of the tendon demonstrated using the VAS scale. Similar findings were reported by Singaraju et al. [36], who used VAS score for the assessment of painful conditions in the course of the
LHBT tendinopathy. Other authors reported that the LHBT tendinopathy related symptoms are night-pains, often expanding to arm and radiating longitudinally; however, in our group this symptom was seen in fewer patients than in other authors' data [3].

As mentioned previously, patient-reported symptoms are not specific to LHBT lesions; unfortunately the same can be said about numerous clinical tests for the LHBT [40]. The most accurate and the simplest test is the tenderness over the bicipital groove. In our study tenderness over the bicipital groove was negative for 2 subjects - one patient had a low VAS score (5 points) and absence of neovascularisation process, another patient had a complete LHBT rupture and probably it confused the clinical examination; this patient also had high neovascularisation grade and VAS scores (grade 3 and 8 points, respectively). Moreover, 6 patients with no neovascularisation reported anterior shoulder pain (VAS score 5-9) and 5 of them had bicipital groove tenderness. Although these patients had concurrent intra-articular lesions: RCTs, subacromial impingement and acromio-clavicular joint dysfunction, we cannot unequivocally explain the discrepancy between clinical and microscopic findings in these cases. The bicipital groove is covered by other structures and lies in the proximity of supraspinatus tendon and subscapularis tendon insertion [37]. Additionally, examination of obese or muscled patients is becoming difficult. All mentioned features complicate the physical examination [37].

One of possible explanations of pain related to LHBT lesions comes from the fact that advanced tendinopathy is usually associated with rich angiofibroblastic reaction accompanied by neoinnervation [39]. Nerve endings secrete substances named neurotransmitters that are responsible for pain sensation. It is thought that chronic painful disorders are a result of uncontrolled release of the neurotransmitters which may lead to disruption of the homeostasis [25]. The presence of the neurotransmitters and their connections with pain were demonstrated in other tendons $[2,5]$. Nociceptive nerve endings were often found close to vessels and pain mediators, e.g. substance $P$ and CGRP appeared in proximity to blood vessels [26].

Similarly, Singaraju et al. [36] who examined formation of new nerves in LHBT tendinopathy using markers of substance $P$ and CGRP did not demonstrate a clear relationship between microscopic and clinical findings. Their findings are consistent with our study and could suggest that pain is related to 
functional changes in these endings, and not directly related to the number of the endings. This also indicates, that the LHBT tendinopathy is not a sole cause of the anterior shoulder pain and more structures may be involved in this process.

Chronic tendinopathy is widely described as a painful process, with a new vessels expansion in a failed attempt to regenerate the tendon, which leads to an increased number of disorganised capillaries. In order to allow for a clear visualisation of this process, we decided to conduct immunohistochemical staining CD31 and CD34. This method demonstrated continuous and regular expression of both markers, which did not appear beyond vessels, allowed us to evaluate in-growth of the new capillary vessels and proved to be useful as markers of neovascularization process in tendons' pathology.

Our control group of healthy semitendinosus tendons did not revealed neovascularisation process and on the other hand, a control group of vascular tumours specimens was characterised by rich and clear CDs immunostaining. Although Pusztaszeri et al. [29] described heterogeneous expression of CD31 and CD34 in different tissues of the human body and demonstrated that it may be related to ageing, we did not demonstrate such an effect in our material.

Feasibility of these markers in visualisation of neovascularisation in tendinopathies was also demonstrated by other authors. Kragsnaes et al. [20] successfully used CD34 staining in specimens obtained from Achilles tendons affected by chronic tendon disease and tendons obtained from healthy volunteers. They have noted that immune-competent cells prevailed in the majority of the specimens which were obtained from degenerated tendons. Fields of the positive CD34 reaction were present predominantly in tendons affected by tendinopathy. Another study by Xu et al. [39] reported the presence of positive reaction of the endothelial cell markers in rotator cuff tendons affected by chronic disorder and the negative reaction in healthy subscapularis tendons. It must be however emphasized that other neovascularisation markers, such as factor VIII-related antigen, CD105, smooth muscle actin have also been proposed [10, 14].

The main limitation to our study was investigation of patients without isolated LHBT tendinopathy. The LHBT tendinopathy is usually associated with at least one important shoulder pathology. Tendons obtained from humans usually represent advanced pathological process and there is limited access to patients in the initial phase of the disorder. It must be however underlined that such limitations were also reported by other authors, and currently no other methods are available to study the role of neoangiogenesis in humans [36]. Another weakness comes from the fact that the group of patients included in this study was relatively small and non-homogenous. Additionally, since we did not harvest parts of the tendon adjacent to its insertion, we were unable to study neovascularisation in this part, which could potentially be characterised by a denser network of capillaries arising from the supraglenoid tubercle.

\section{CONCLUSIONS}

Our study confirmed that neovascularisation is a common process in the course of the LHBT tendinopathy. We demonstrated that immunohistochemical techniques using CD31 and CD34 markers allow for a clear visualisation of newly formed capillaries. However, although it is thought that new vessels formation is accompanied by the neural tissue ingrowth and release of the neurotransmitters, we were unable to demonstrate a relationship between microscopic findings and pain level in our patients. This suggests that anterior shoulder pain is a complex phenomenon, which involves pathologies of the LHBT and concomitant structures. Since we demonstrated that clinical examination may not be sufficient to precisely diagnose LHBT tendinopathy, we are strongly convinced that subsequent studies are needed to gain insight into the relationship between tendinopathy, neovascularisation and pain.

\section{REFERENCES}

1. Abate M, Silbernagel KG, Siljeholm C, et al. Pathogenesis of tendinopathies: inflammation or degeneration? Arthritis Res Ther. 2009; 11(3): 235, doi: 10.1186/ar2723, indexed in Pubmed: 19591655.

2. Ackermann PW. Neuronal regulation of tendon homoeostasis. Int J Exp Pathol. 2013; 94(4): 271-286, doi: 10.1111/iep.12028, indexed in Pubmed: 23718724.

3. Ahrens PM, Boileau P. The long head of biceps and associated tendinopathy. J Bone Joint Surg Br. 2007; 89(8): 1001-1009, doi: 10.1302/0301-620X.89B8.19278, indexed in Pubmed: 17785735.

4. Alfredson $\mathrm{H}$, Bjur D, Thorsen K, et al. High intratendinous lactate levels in painful chronic Achilles tendinosis. An investigation using microdialysis technique. J Orthop Res. 2002; 20(5): 934-938, doi: 10.1016/ S0736-0266(02)00021-9, indexed in Pubmed: 12382956.

5. Alpantaki K, McLaughlin D, Karagogeos D, et al. Sympathetic and sensory neural elements in the tendon of the long head of the biceps. J Bone Joint Surg Am. 2005; 87(7): 1580-1583, doi: 10.2106/ JBJS.D.02840, indexed in Pubmed: 15995126.

6. Andrzejewski W, Kassolik K, Dziegiel P, et al. Massage may initiate tendon structural changes: a preliminary study. In Vivo. 2015; 29(3): 365-370, indexed in Pubmed: 25977382. 
7. Andrzejewski W, Kassolik K, Kobierzycki C, et al. Increased skeletal muscle expression of VEGF induced by massage and exercise. Folia Histochem Cytobiol. 2015; 53(2): 145-151, doi: 10.5603/FHC. a2015.0013, indexed in Pubmed: 26150286.

8. Aström M, Rausing A. Chronic Achilles tendinopathy. A survey of surgical and histopathologic findings. Clin Orthop Relat Res. 1995(316): 151-164, indexed in Pubmed: 7634699.

9. Aström M, Westlin N. Blood flow in chronic Achilles tendinopathy. Clin Orthop Relat Res. 1994(308): 166-172, indexed in Pubmed: 7955678.

10. Bjur D, Alfredson H, Forsgren S. The innervation pattern of the human Achilles tendon: studies of the normal and tendinosis tendon with markers for general and sensory innervation. Cell Tissue Res. 2005; 320(1): 201-206, doi: 10.1007/s00441-004-1014-3, indexed in Pubmed: 15702331.

11. DeYoung BR, Wick MR, Fitzgibbon JF, et al. CD31 immunoreactivity in mesenchymal neoplasms of the skin and subcutis: report of 145 cases and review of putative immunohistologic markers of endothelial differentiation. J Cutan Pathol. 1995; 22(3): 215-222, indexed in Pubmed: 7593814.

12. Ditsios K, Agathangelidis F, Boutsiadis A, et al. Long head of the biceps pathology combined with rotator cuff tears. Adv Orthop. 2012; 2012: 405472, doi: 10.1155/2012/405472, indexed in Pubmed: 23209915.

13. Fenwick SA, Hazleman BL, Riley GP. The vasculature and its role in the damaged and healing tendon. Arthritis Res. 2002; 4(4): 252-260, doi: 10.1186/ar416, indexed in Pubmed: 12106496.

14. Florence ME, Massuda JY, Bröcker EB, et al. Angiogenesis in the progression of cutaneous squamous cell carcinoma: an immunohistochemical study of endothelial markers. Clinics (Sao Paulo). 2011; 66(3): 465-468, indexed in Pubmed: 21552674.

15. Gisslén K, Gyulai C, Nordström P, et al. Neovascularisation and pain in jumper's knee: a prospective clinical and sonographic study in elite junior volleyball players. Br J Sports Med. 2005; 39(7): 423-8; discussion 423, doi: 10.1136/bjsm.2004.013342, indexed in Pubmed: 15976162.

16. Harpreet SS, El Rassi G, Bahk MS, et al. Physical examination for partial tears of the biceps tendon. Am J Sports Med. 2007; 35(8): 1334-1340, doi: 10.1177/0363546507300058, indexed in Pubmed: 17369556.

17. Hitchcock HH, Bechtol CO. Painful shoulder; observations on the role of the tendon of the long head of the biceps brachii in its causation. J Bone Joint Surg Am. 1948; 30A(2): 263-273, indexed in Pubmed: 18912289.

18. Joseph M, Maresh CM, McCarthy MB, et al. Histological and molecular analysis of the biceps tendon long head post-tenotomy. J Orthop Res. 2009; 27(10): 1379-1385, doi: 10.1002/jor.20868, indexed in Pubmed: 19340876.

19. Khan KM, Cook JL, Bonar F, et al. Histopathology of common tendinopathies. Update and implications for clinical management. Sports Med. 1999; 27(6): 393-408, indexed in Pubmed: 10418074.

20. Kragsnaes MS, Fredberg U, Stribolt $K$, et al. Stereological quantification of immune-competent cells in baseline biopsy specimens from achilles tendons: results from patients with chronic tendinopathy followed for more than 4 years. Am J Sports Med. 2014; 42(10): 2435-2445, doi: 10.1177/0363546514542329, indexed in Pubmed: 25081311.

21. Lewis JS, Raza SA, Pilcher J, et al. The prevalence of neovascularity in patients clinically diagnosed with rotator cuff tendinopathy. BMC Musculoskelet Disord. 2009; 10: 163, doi: 10.1186/14712474-10-163, indexed in Pubmed: 20025761.

22. Maffulli N, Longo UG, Franceschi F, et al. Movin and Bonar scores assess the same characteristics of tendon histology. Clin Orthop Relat Res. 2008; 466(7): 1605-1611, doi: 10.1007/s11999-0080261-0, indexed in Pubmed: 18437501.
23. Matthews JP. Vascular changes in flexor tendons after injury and repair: an experimental study. Injury. 1977; 8(3): 227-233, indexed in Pubmed: 328391.

24. Molloy TJ, Wang Y, Murrell GAC, et al. The roles of growth factors in tendon and ligament healing. Sports Med. 2003; 33(5): 381-394, indexed in Pubmed: 12696985.

25. Murthi AM, Vosburgh CL, Neviaser TJ. The incidence of pathologic changes of the long head of the biceps tendon. J Shoulder Elbow Surg. 2000; 9(5): 382-385, doi: 10.1067/mse.2000.108386, indexed in Pubmed: 11075320.

26. Ohberg L, Lorentzon R, Alfredson H. Neovascularisation in Achilles tendons with painful tendinosis but not in normal tendons: an ultrasonographic investigation. Knee Surg Sports Traumatol Arthrosc. 2001; 9(4): 233-238, doi: 10.1007/s001670000189, indexed in Pubmed: 11522081.

27. Pisacane AM, Picciotto F, Risio M. CD31 and CD34 expression as immunohistochemical markers of endothelial transdifferentiation in human cutaneous melanoma. Cell Oncol. 2007; 29(1): 59-66, indexed in Pubmed: 17429142.

28. Poblet E, Gonzalez-Palacios F, Jimenez FJ. Different immunoreactivity of endothelial markers in well and poorly differentiated areas of angiosarcomas. Virchows Arch. 1996; 428(4-5): 217-221, indexed in Pubmed: 8764929.

29. Pusztaszeri MP, Seelentag W, Bosman FT. Immunohistochemical expression of endothelial markers CD31, CD34, von Willebrand factor, and Fli-1 in normal human tissues. J Histochem Cytochem. 2006; 54(4): 385-395, doi: 10.1369/jhc.4A6514.2005, indexed in Pubmed: 16234507

30. Rees JD, Stride M, Scott A. Tendons--time to revisit inflammation. Br J Sports Med. 2014; 48(21): 1553-1557, doi: 10.1136/ bjsports-2012-091957, indexed in Pubmed: 23476034.

31. Rees JD, Wilson AM, Wolman RL. Current concepts in the management of tendon disorders. Rheumatology (Oxford). 2006; 45(5): 508-521, doi: 10.1093/rheumatology/kel046, indexed in Pubmed: 16490749.

32. Richards HJ. Repair and healing of the divided digital flexor tendon. Injury. 1980; 12(1): 1-12, indexed in Pubmed: 7203616.

33. Riley G. Tendinopathy - from basic science to treatment. Nat Clin Pract Rheumatol. 2008; 4(2): 82-89, doi: 10.1038/ncprheum0700, indexed in Pubmed: 18235537.

34. Riley $G$. The pathogenesis of tendinopathy. A molecular perspective. Rheumatology (Oxford). 2004; 43(2): 131-142, doi: 10.1093/ rheumatology/keg448, indexed in Pubmed: 12867575.

35. Russell Jones R, Orchard G, Zelger B, et al. Immunostaining for CD31 and CD34 in Kaposi sarcoma. J Clin Pathol. 1995; 48(11): 1011-1016, indexed in Pubmed: 8543622.

36. Singaraju VM, Kang RW, Yanke AB, et al. Biceps tendinitis in chronic rotator cuff tears: a histologic perspective. J Shoulder Elbow Surg. 2008; 17(6): 898-904, doi: 10.1016/j.jse.2008.05.044, indexed in Pubmed: 18786837

37. Snyder GM, Mair SD, Lattermann C. Tendinopathy of the long head of the biceps. Med Sport Sci. 2012; 57: 76-89, doi: 10.1159/000328880, indexed in Pubmed: 21986047.

38. Streit JJ, Shishani $Y$, Rodgers $M$, et al. Tendinopathy of the long head of the biceps tendon: histopathologic analysis of the extraarticular biceps tendon and tenosynovium. Open Access J Sports Med. 2015; 6: 63-70, doi: 10.2147/OAJSM.S76325, indexed in Pubmed: 25792859

39. $\mathrm{Xu} Y$, Bonar F, Murrell GAC. Neoinnervation in rotator cuff tendinopathy. Sports Med Arthrosc Rev. 2011; 19(4): 354-359, doi: 10.1097/ JSA.0b013e318229d7e3, indexed in Pubmed: 22089285.

40. Zabrzyński J, Zabrzyńska A, Grzanka D. Tendinopathy - a disease of tendons. J Orthop Trauma Surg Related Res. 2016; 3(40): 024-030. 